\title{
CONTROLLED MULTIFOCAL FRONTAL LEUCOTOMY FOR PSYCHIATRIC ILLNESS
}

\author{
BY \\ H. J. CROW, R. COOPER, and D. G. PHILLIPS \\ From the Burden Neurological Institute, and Frenchay Hospital, Bristol
}

There have been two important developments in the surgical treatment of mental disorders during the last 12 years, and one of these is in the selection of patients. No longer is the chronic schizophrenic the principal type of patient selected for cerebral surgery. The patients now considered for surgical treatment are usually chronic depressives and those with anxiety states, and obsessional syndromes whose symptoms have proved intractable. These cases include young and intelligent people, some with potentially promising futures and many with heavy responsibilities. In such cases it is particularly important to preserve as much as possible of intellectual capacity, sense of responsibility, and social judgment.

The need for more discriminating intervention in cerebral function has been reflected in the variations in operative techniques used in cerebral surgery in recent years. The second important change, then, has been the introduction of methods for the selection and limitation of the site of the destruction so as to do the least damage that will bring significant and prolonged relief of symptoms to the patient. Many attempts have been made to achieve the goal of most relief with least damage. Frontal topectomy was described by Pool (1949), anterior cingulectomy by Whitty, Duffield, Tow, and Cairns (1952), electro-coagulation of limited areas in the thalamic nuclei by Spiegel and Wycis (1949), orbital undercutting by Scoville (1949), frontal electro-coagulation by Grantham (1951), bimedial leucotomy by Greenblatt and Solomon (1952), transorbital leucotomy by Freeman (1948), lower quadrant leucotomy by Thorpe and Hardman (1952), and rostral leucotomy by McKissock (1951).

White, Sweet, and Hackett (1959) have described a method of leaving coagulating electrodes in the frontal lobes of brain for prolonged periods to allow the lesion to be enlarged at a later date if the clinical state indicates the need. They used one or two large electrodes and coagulated by diathermy. This technique was used in cases of intractable pain (usually caused by cancer).
These methods have two limitations: first, however well controlled the lesion is anatomically, they are functionally 'blind' and irreversible; second, being essentially acute operations it is difficult to grade the lesion to match the illness of the patient. Greater delicacy and control of coagulation of frontal white matter can be achieved by using a large number of small, chronically indwelling, intracerebral electrodes.

\section{Technique}

Twenty-four to 34 electrodes $(4 \mathrm{~mm}$. long, $0.2 \mathrm{~mm}$. diameter) are inserted in each frontal lobe. Those lying in white matter are identified by stimulation. Through each one of these electrodes in turn a small direct current is passed, which causes a temporary suppression of the physiological function of the axons surrounding the electrode, in effect performing a minute, focal, reversible leucotomy. This may be seen as a temporary change in the clinical state of the patient. In this way the potential clinical effect of electrolysis of many small regions can be assessed. The progressive electrolysis of sites thus selected is undertaken over a period of weeks or months until a favourable clinical state has been attained.

\section{Methods}

Electrodes.-There are two important factors which determine the choice of electrode material for this method of treatment. The first is electro-chemical inertness. Since the electrodes are to remain implanted in the brain for months, there must be no toxic effects due to the metal. Fischer, Sayre, and Bickford (1957) showed that copper and silver produced local necrosis of brain tissue but that stainless steel was inert. We have found that gold wire is also inert. The second factor to consider is the current-carrying capacity of the electrode. MacIntyre, Bidder, and Rowland (1959) have shown that for small currents the size of an electrolytic lesion (at the anode) is directly related to the quantity of electricity (current $x$ time) passed through the electrode. While the current is flowing through the electrode, considerable erosion of the electrode can take place, especially if the electrode is made from stainless steel (Loucks, Weinberg, and Smith, 1959).

Experiments with various electrode materials in vitro have shown that electrodes made from 18/8 stainless 
FIG. 1b.-Photograph of introducer needle through which the electrodes are passed. The stillette has been partly withdrawn. The adjustable clip on the needle butts against the stereotaxic frame when the tip of the needle has reached a pre-determined depth in the brain.

steel 0.005 in. diameter $(125 \mu)$ with $4 \mathrm{~mm}$. bared will erode away if $10 \mathrm{~mA}$ current is passed for 45 seconds (450 milli-coulombs). With 36 gauge ( 0.0076 in., $190 \mu)$ Staybrite steel wire $10 \mathrm{~mA}$ ( 2400 milli-coulombs) can be passed for four minutes in physiological saline. The size of the lesion produced by a fixed quantity of electricity is also dependent upon the electrode material. MacIntyre et al. have shown that for low quantities of electricity (40 milli-coulombs), the volume index in c. $\mathrm{mm}$. (idealized volume of lesion) is $3 \cdot 1$ for gold, $3 \cdot 1$ for copper, $2 \cdot 4$ for silver, $1 \cdot 3$ for platinum, and $0 \cdot 76$ for stainless steel.

The conclusion is that for these purposes the best electrode metal is gold. We are now using 38 gauge $(0.006$ in., $150 \mu)$ fine gold wire insulated with Simgold enamel.* We have found that $10 \mathrm{~mA}$ current passed through a standard gold electrode $(4 \mathrm{~mm}$. long, about 2 sq. mm. area) for three minutes produces a macroscopic lesion, approximately $5 \mathrm{~mm}$. long and $3 \mathrm{~mm}$. diameter, in fresh cadaver brain. Gold electrodes of these dimensions show negligible signs of erosion. At the time of treatment of some of the patients described in this report, the experiments described above had not been completed and stainless steel, and on one occasion silver, electrodes have been used.

The electrode sheaves are spun on a special instrument, using six gold wires round a stainless steel stiffening wire $(36 \mathrm{~g}$.). The gold wires are cut to different lengths so that there are $8 \mathrm{~mm}$. between the tips of neighbouring electrodes and $4 \mathrm{~mm}$. of the insulation is stripped from the end of each gold wire (Fig. 1).

Insertion of Electrodes.- - Our technique is based on the method originally used by Dodge, Holman, SemJacobsen, Bickford, and Petersen (1953).

A simple stereotactic frame was built in our workshop, and this has been found to be precise enough for our purpose. The electrodes are introduced, under a general anaesthetic, through bilateral frontal burr holes in the coronal suture, about $3 \mathrm{~cm}$. from the mid-line.

*F. D. Sims Ltd., Ramsbottom, Lancs, England.
Four or five sheaves of electrodes (six in each sheaf) are placed fan-wise in the coronal plane. Originally the fan of electrodes was spread widely in the frontal lobes, just behind the tips of the ventricles, but from experience with the first cases the disposition of the electrode sheaves + has been changed so as to narrow the fan and move it backwards into the coronal plane, 0.5 to $1.0 \mathrm{~cm}$. anteriō to the anterior clinoids (Figs. 2 and 3 ).

After the operation the ends of the electrode wires ot:side the scalp (coded for identification) are soldered to $z$ thin insulated tinned copper wires terminated by tw으응 34-way subminiature sockets. $\dagger$ If normal soldering ter peratures are used the gold alloys with the solder amd $\bar{\sigma}$ disappears into solution. This is prevented by reducimg the temperature of the soldering iron to just above the melting point of solder. The sockets are secured to

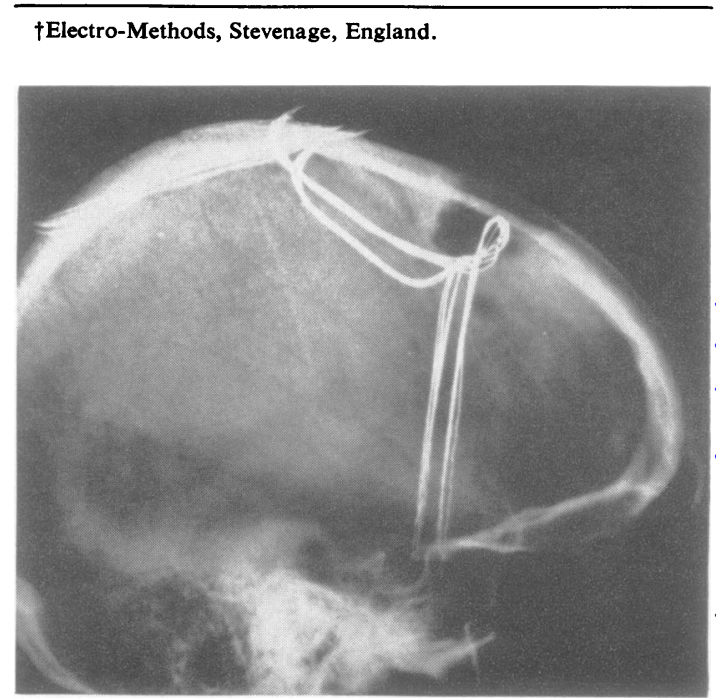

Fig. 2.-Lateral view of the electrode sheaves. 


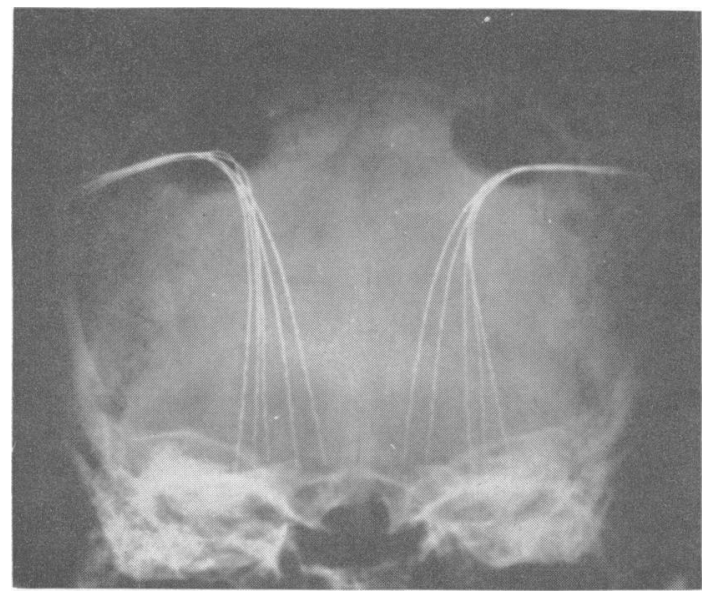

Fig. 3a

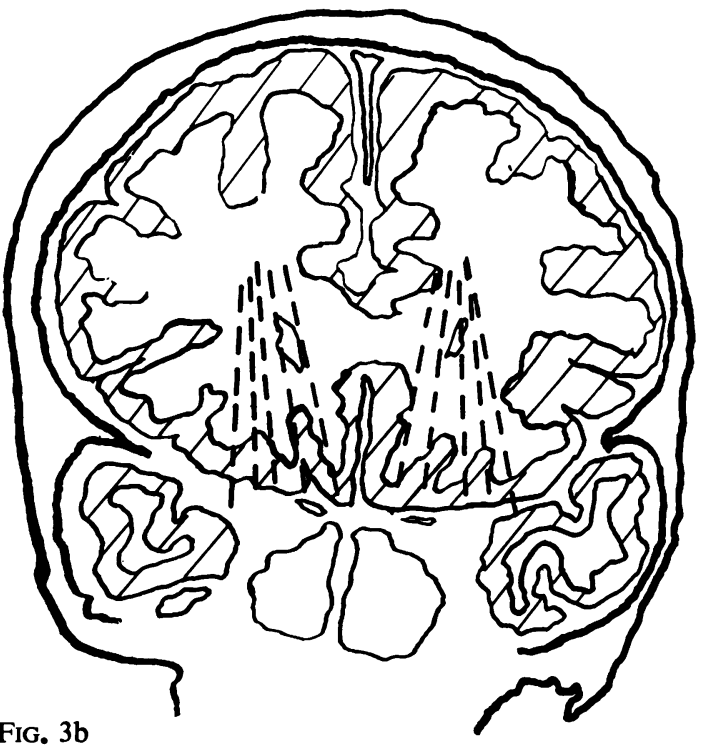

Fig. 3a.-Antere-posterior view of the electrode sheaves.

(b) Diagram showing the disposition of the individual electrodes in the brain tissue. The cross hatched portion represents the grey matter.

(The section is taken from Cranio-Cerebral Topometry in Man by Delmas and Pertuiset, published by Masson \& Cie, Paris, and Blackwell Scientific Publications, Oxford.)

loops of stainless steel wire left in the skull using loops of cotton tape. The electrode wires are taped together with polythene tape, and the bundle fastened to the scalp with surgical strapping. Within three or four days of the operation the patient is up and about. Testing and treatment can be started after about seven days.

Testing and Treatment.-This is done with the patient fully conscious lying on a bed. While testing and treatment are being performed according to a pre-arranged plan, the clinician sits by the bedside talking to the patient, exploring the clinical psychiatric picture, asking for subjective impressions from the patient, and noting any objective changes in the patient's state. Throughout the procedure he holds in his hand a switch which can cut out the passage of all current, if this should seem necessary or desirable (Fig. 5).

Identification of Grey and White Matter.-One of the important advantages of using multiple indwelling electrodes is that lesions can be restricted to white matter, thus avoiding the risk of producing cortical scars which might prove to be epileptogenic. Identification of electrode positions in relation to white and grey matter cannot be achieved by stereotactic control because of the great variation in convolutional pattern between individuals. The fine details of location are best established by functional observations after the electrodes have been in position for some days. In the first cases measurements were made of the passive electric impedance of all the electrodes: this was found to vary from electrode to electrode in a way suggesting that those in grey matter offered a lower impedance than those in white matter. The impedance differences were not very great, however, and a more trustworthy criterion was found to be the propensity of the brain tissue around any particular electrode to develop and sustain an organized afterdischarge when stimulated electrically.

Physiological observations have shown that stimulation at cathode electrodes in cortical grey matter can evoke a characteristic local after-discharge above a certain threshold stimulus intensity which is remarkably constant when the other stimulus parameters-stimulation period, pulse duration, and frequency-are fixed (Fig. 4). This is the type 2 after-discharge, defined by Chang (1959) as involving the activity of closely situated neurons. On the other hand, stimulation at electrodes in white matter has no such effect at intensities several times greater than the threshold for grey matter. This effect has been confirmed in our observations: stimulation at electrodes likely to be in frontal white matter has never evoked an after-discharge, while exploration of those in regions likely to be involved in convolutions of grey matter have yielded the patterns of after-discharge distribution to be expected from anatomical diversity (Fig. 3B).

In order to establish the range of variation of electrical threshold in normal cortex a number of measurements have been made of cortical excitability in various regions of the brain in these and other types of patient. With electrodes in sensory, e.g., visual, cortex subjective sensations as well as after-discharges can be used as criteria of response (alterations in oxygen availability have also been found useful in this connexion, see Cooper, Crow, Walter, and Winter, 1961). Using a constant-voltage, i.e., low impedance, stimulator, the threshold for production of an organized local after-discharge in normal cortex is about 4 volts when the pulse width is fixed at $400 \mu \mathrm{sec}$. at a pulse rate of 40 per sec. and a stimulation period of 10 seconds. Bipolar stimulation between adjacent electrodes in a sheaf has been used throughout to limit current spread. When electrode pairs in white matter are 


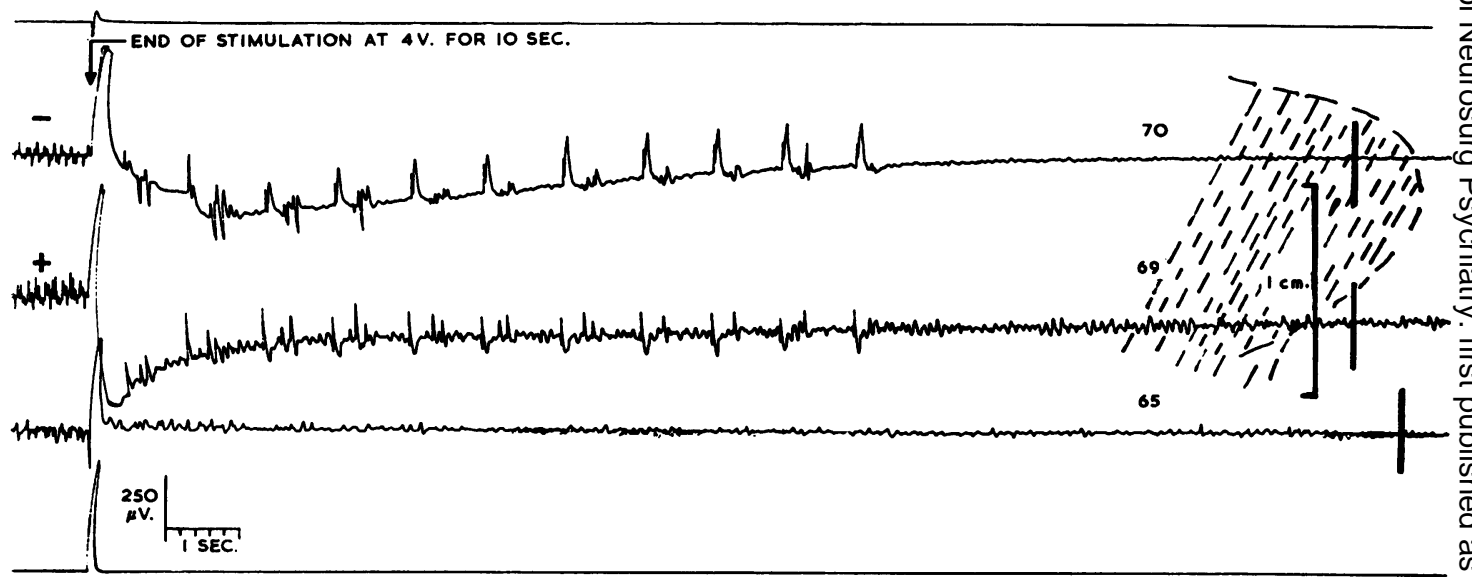

FIG. 4.-Localized after-discharge following stimulation of electrode No. 70 in convolution of grey matter deep in right lateral frontal lobe. $\vec{\varnothing}$ Stimulus pulse duration $400 \mu \mathrm{sec}$., frequency 40 per sec. The typical rhythm, restriction, and abrupt termination distinguish between -1 electrodes in such regions and those in white matter (electrode 65 , channel 3 ) where stimulation at much higher voltages evokes no after- $\vec{\omega}$ discharge.

tested, voltages two or three times greater than this produce no after-discharge.

Exploration of the many therapeutic electrodes in the frontal lobes occasionally show after-discharge thresholds of about 8 volts; these are usually adjacent to electrodes showing a lower threshold and are considered to be close to the border between white and grey matter.

Although the waveform and persistence of cortical after-discharges often recall the 'seizure patterns' considered as characteristic of epilepsy in scalp E.E.G. records, this resemblance is misleading since the discharges evoked by focal stimulation involve only very small volumes of cortical tissue-about $0.5 \mathrm{cub}$. cm.and the normal intrinsic electrical activity continues unimpeded in regions only a few millimetres away from the stimulating cathode. The extreme restriction of these effects is of considerable physiological interest and importance and is corroborated by the subjective sensations, which are minimal, and often totally absent. Even when electrodes in visual cortex are stimulated, the patients have reported only localized phosphenes, and have not experienced any disturbing sensations.

Using these methods it is possible to chart the functional position of all the electrodes in a few hours, and from this information can be derived a map of the cerebral regions involved showing the distribution of grey sulci which often extend deep into the frontal lobes.

Polarization and Coagulation.-The electrolytic lesions are made using a constant current device similar to that described by Fleming (1957), The direct current can be pre-set to any value from 0 to $20 \mathrm{~mA}$, and the time of rise and fall, to and from this value, is variable from 0 to 60 sec. The electrode around which the lesion has to be made is connected to the positive terminal of the constant current device, and the negative terminal is connected to a large electrode strapped to the forearm.

With the patient fully conscious, and lying comfortably on a bed, low currents ( $1 \mathrm{~mA}$ for $15 \mathrm{sec}$.) are passed in turn through each of the electrodes lying in frontal white? matter. The clinical and physiological state of the patient is observed for some minutes after each polarization. $\rightarrow$ We have found that it is possible to relieve symptoms when certain electrodes are 'polarized' in this way, a卬ि that this relief will persist for periods varying from $\$ 5$ 음 minutes to 24 hours. This temporary relief may be dọeto very small amounts of permanent damage, with other $z$ fibres later taking over the function of those destroyed; or it may be due to temporary electrotonic change \&ु fibres which subsequently recover. After the symptoins have returned, polarization of the same electrode at the same or a slightly higher current value (for the same $\vec{\theta}$ length of time) will produce temporary relief again. Thus is achieved a reversible focal functional leucotomy.

In order to coagulate the tissue in regions where temporary relief has been obtained, progressively larger quantities of electricity are passed through the electrode. $\bar{\partial}$ The steps are usually $2 \mathrm{~mA}$ for $15 \mathrm{sec}$., $5 \mathrm{~mA}$ for $15 \mathrm{sec}$, , $10 \mathrm{~mA}$. for $60 \mathrm{sec}$, and finally $20 \mathrm{~mA}$ for $180 \mathrm{sec}$. During $\triangle$ the polarization and coagulation sessions the electrocardiogram, psychogalvanic reflex, respiration, and $\overrightarrow{\bar{O}}$ electro-encephalogram from the region being coagulated $\exists$ are recorded continuously. In a session lasting about one $?$ hour, up to six electrodes can be treated in this way. Fig. 5 shows the scene immediately after treatment by coagulation of Case 1.

\section{Results}

The first five cases treated by this method are now described.

Case 1.-A married woman of 36 had the first of 윽 innumerable, unaccountable panic attacks 18 years ago. Twelve years ago she developed an obsessional fear that she would kill her infant daughter. Six years ago she $N$ developed an obsessional concept of a stereotyped devil $\Omega$ (with horns, tail, and cloak) in her head, telling her to $N$ 


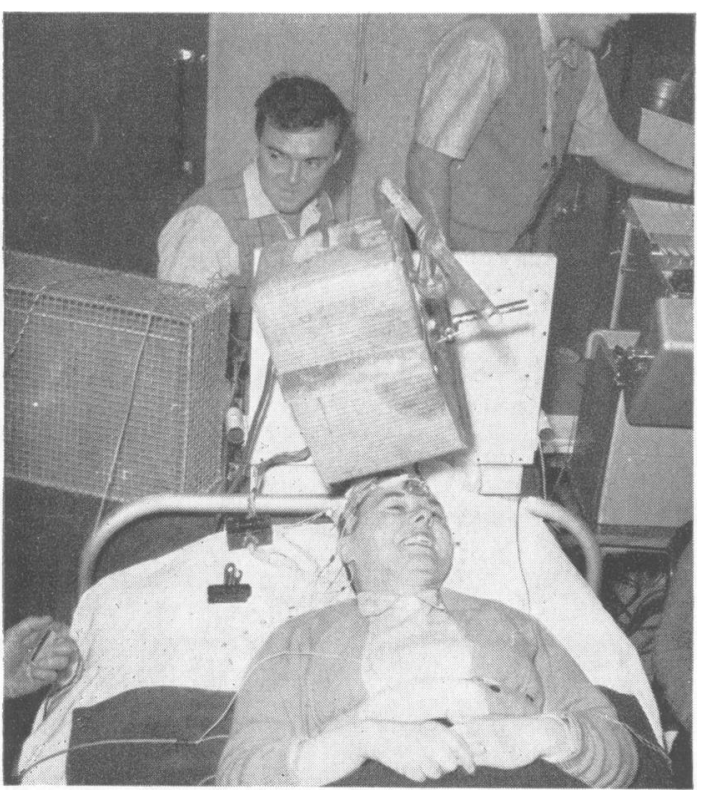

Fig. 5.-Case 1 immediately after the final coagulation of frontal white fibres.

kill her daughter and commit suicide. Although she had insight into the delusory nature of her devil, she was terrified by him. Periodically these symptoms, which were almost constant, drove her to depression and despair. She had had psychotherapy in three hospitals, several courses of E.C.T., and many sedatives and tranquillizers. The only treatment which ever helped was the E.C.T., which relieved only the depression. At the time of operation she had been in hospital for two years, and was quite incapable of leading a normal life.

After the insertion of the intracerebral electrodes, her symptoms of fear and tension were consistently, although temporarily, relieved by polarization of two of the medial electrodes lying about mid-way between the vertex and the floor of the anterior fossa of the skull. Another effect of polarization was to banish the 'devil', but he returned later in modern dress, with a more benign attitude. Coagulation of four points in the frontal lobes produced complete relief of symptoms, and the wires were removed two and a half weeks later.

This remission, however, was shortlived. Within the following 10 weeks she recapitulated the whole complex sequence of events of her 18 years' history of illness. On the assumption that the suppression of abnormal function had been inadequate and in view of the dramatic relief obtained earlier, it was decided to repeat and extend the procedure. A second set of electrodes was inserted in approximately the same position as before. On this occasion the wires were left in situ for three months, thus giving a longer period to test the treatment. The lesions were extended and increased in number by stages to eight in each lobe. Again an excellent mental state of calmness without obsessional thoughts or 'devil' resulted. As her symptoms declined, so she acquired a new 'warmth' of feeling for her daughter.

Her condition has steadily improved, and she has now been home for two years living a normal life. There has been no euphoria and no irresponsibility or overconfidence. She has returned to the hospital several times to help and comfort and encourage other patients, and has offered pertinent observations about problems of inter-personal relationships amongst patients in the wards.

Case 2.-A married woman of 60 had a lifelong history of migraine, with a serious functional (hysterical) extension of the pains. Over the years she had had many investigations and treatments. In the last year she had become desperate and suicidal. Electro-convulsive treatment had given only shortlived relief. Electrodes were inserted in the frontal lobes. This patient was treated almost concurrently with the first case. As in the first case, there was an excellent recovery after coagulation in four regions, and the electrodes were removed. But within a few weeks she had relapsed so seriously that an immediate modified 'rostral' type of leucotomy was performed. She has remained quite well in the past two years although she sometimes talks of the head pains.

Case 3. - A married woman of 34 had felt since childhood an obsessional need to think out in detail everything she was going to do, and to consider in detail everything that she had done. If she is interrupted in her prolonged cogitations, which she calls 'my thinks', or is otherwise unable to complete them, she becomes assailed by feelings of guilt and fear. While she was single, she had been able to indulge her obsessional need for 'thinks', by living a slow and simple life. But when a husband, and a home, and then a child were added to her life, she found the day too short for all the necessary 'thinks'. She broke down in a state of terror, exhaustion, and depression. Electro-convulsive therapy relieved the depression but made no difference to the other symptoms. Three stays in hospital were necessary in the 18 months before the insertion of frontal electrodes.

By the time the electrodes were inserted she had been in hospital for some weeks, and in the simple life there, she had lost all her fear and tension. She was calmly performing her daily 'thinks'. Therefore, apart from her 'thinks' she had no symptoms when treatment was being conducted, and the amount of destruction required could not be tested by clinical emotional response.

Sixteen lesions (all smaller than those made in more recent cases) were made in the frontal lobes and the wires were then removed.

She was happy and relaxed, but was indulging in her life-long practice of 'thinks', though she could easily be distracted from them. She seemed to be progressing steadily, if slowly, in abolishing one set of 'thinks' after another. However, after discharge from hospital, she moved into a new house, and the stress of a new set of 'thinks' built up again. She is at present back in hospital, and has severe panics when the 'thinks' are prevented.

This case is a failure. In the light of subsequent experience, not enough damage was done to the 
system associating the emotion of fear with conceptualization. This case was treated during the early stages of development when we were overoptimistically conservative about the amount of interference with frontal lobe function needed to relieve such symptoms in patients subjected to continuing social stress.

Case 4.-A widow of 59 had suffered an uninterrupted anxiety-depressive illness for 10 years. She had always reacted to stress with excessive anxiety. During the 10 years of her illness she had had three courses of E.C.T. without significant benefit. She had had stimulant and sedative and tranquillizing drugs. She had been treated by psychotherapy. She had two hospital admissions before leucotomy was offered her.

After treatment by this method of focal frontal leucotomy (eight focal lesions in each frontal lobe) she was completely relieved of her symptoms. We think we did a little too much damage in this case. She lost some intellectual capacity as measured by Wechsler tests, the score dropping from 114 to 109 , and the world was a little too 'rosy' for her. But she is fully responsible socially, and considerate of other people's feelings. She has remained very well in the year since treatment.

Case 5.-A fit married man of 46 is intelligent, and, following a course of psychotherapy six years ago, has very good insight. All his life he has been afraid of people, and has lived a secluded personal life, although he became a technical sergeant in the R.A.F., and is married and has two children. His internal tensions and fears had become steadily more intense in recent years, and he finally had to give up work because he could not talk to his colleagues in the office. Drugs failed to help him. There did not seem to be any indication for E.C.T. He was treated by this method (16 focal lesions in each frontal lobe) and has made an excellent recovery. Two months after the withdrawal of the electrodes, he returned to work in the Civil Service. His I.Q., as measured by Wechsler tests, had increased from 122 to 132 after treatment. He has a proper balanced optimism about the future. There has been an increase in the warmth of feeling between him and his wife and children. In the first five months since the treatment, his mental state has steadily improved. The constant feelings of fear and tension disappeared early in his treatment. The abnormalities in his conceptualizing about his relationships with other people in particular with his office colleagues, have subsequently been progressively changing towards normality.

\section{Discussion}

At first sight, the method described has certain unfavourable features. Infection is the first obvious danger to consider. This technique for implanting electrodes has been used in several hundreds of cases in America and Europe for a period of seven years. During that time there have been reports of only two cases of meningitis, and one of an infection of unspecified type.
The second danger is the production of unwanted trauma to the brain by the introduction of the electrodes. Trauma, of course, does occur, but no case has been reported where this has produced clinical signs or symptoms or persistent electrical abnormalities. This danger is naturally set against the benefits expected from the procedure. Needless to say, it is less traumatic than the use of the leucotome.

The third danger is the production of toxic lesions in the brain by the metal of the electrodes or by the insulating material on them. The insulating material, an epoxy resin enamel, is extremely inert. The skin of the scalp heals around the insulated electrode wires without any foreign-body inflammatory reaction. Although base metals are toxic, के stainless steel and gold are harmless, and gold $\vec{\circ}$ anodes are not eroded by the passage of current.

The fourth unfavourable feature is the natural reluctance on the part of physicians and patients to produce such an unlikely and unnatural situation as a man walking around with electrodes in his brain for weeks on end, thus rendering himself liable to have his feelings, moods, and thoughts altered merely by plugging the electrodes into a switch bo $\bar{W}$ and having a switch depressed. We mention this 을 only to report that it is easy to exaggerate thi feature. In practice we have found that our patients $z$ have accepted the situation with extraordinar nonchalance. Friends and relatives have also take it as a matter of course. Other patients in the wards know what is going on but they have not in any was reacted unfavourably. The excellent response of the $\overrightarrow{+} \overrightarrow{\mathscr{\theta}}$ patients to the treatment, of course, impresses the others, who knew them when all the symptoms were present. So gentle and unfrightening is the whole therapeutic process that other patients have been asking to be treated in the same way (Fig. 5).

Usually the patient, although fully conscious, is unaware of the exact time when polarization or electrolysis has occurred; often he does not know that anything has happened. Sometimes, however, he recognizes a sudden reduction of symptoms and smiles in a relieved way. More often the change of mood during a treatment session is gradual. It may be some considerable time after the mood has changed from anxiety to calmness before the patient is properly aware that a change in himself has occurred although it may have been obvious to the observer.

Immediate side-effects have not been severe and the patients have not been disturbed. Headaches during the passage of the coagulating currents have sometimes been reported. Occasionally peculiar head sensations while the current is passing have been described-'like something moving in my head', 
'a crackling noise', 'a squeezed feeling'-but usually the patient talks to the doctor as if nothing unusual were happening.

Delayed side-effects of coagulation have included dull headache during the 24 hours after treatment; nausea some hours after treatment occurred in one case; inattentiveness for some hours up to a few days after treatment has been noticed twice; usually there is a small rise in temperature $\left(0.5\right.$ to $1^{\circ} \mathrm{F}$.) in the evening after treatment. The patients sometimes sleep for an hour or two later in the day of treatment. Apart from the one case of nausea, all patients remained ambulant, ate meals in the dining room and mixed with other patients in an ordinary way.

When these patients wear suitable headgear, the small plugs of the multiple electrode assembly, which they carry strapped to the crown of the head, are quite invisible. It is therefore possible for them to leave the hospital during the course of treatment. They go shopping and sightseeing and spend weekends at home or at work. In these ways, the benefits of treatment can be tested by some of the real environmental stresses of the outside world. If the patient reacts to these stresses by, say, build-up of tension and fear, the existing lesions can be enlarged, or the number of lesions can be increased according to the results of the preliminary polarization. In this way the capacity to build up abnormal emotional states can be reduced appropriately.

This method of treatment of serious mental disorder is more time-consuming than many of the current surgical procedures, and at present requires the attention of more skilled persons but it is still in its development stage; with increasing experience more economical procedures are being evolved. Currently, four patients are under treatment at the same time. In any case, the modification of human feelings, thoughts, and behaviour by interference with brain function cannot be considered as a purely economic proposition; delicacy of control and respect for individual needs are of paramount importance.

This procedure for producing focal frontal leucotomy offers certain advantages:

(1) The situation of the electrodes with respect to white and grey matter can be ascertained. This reduces the risk of lesions being made in cortex and hence the likelihood of epilepsy as a complication of treatment.

(2) Selective polarization of the frontal axons at low energy levels produces a discrete focal leucotomy which is clinically and functionally reversible. This is in effect a trial-and-reversible-error leucotomy. If the trial lesions are found to relieve symptoms and not to produce unwanted effects, the lesions can be made permanent by electrolytic coagulation.
(3) The total number of small discrete lesions can be increased, or the lesions enlarged, progressively over a period of many weeks, which may include a probationary period at home and work. This makes possible a more accurate judgment of the minimum amount of destruction required to relieve a particular patient's symptoms in a normal social environment.

In view of the enormous variation in human personality and brain function it is not strange that a brain lesion which gives one mental patient great benefit may not provide relief for another patient. This multiple electrode method of progressive leucotomy provides a tailor-made pattern of brain lesions to suit the individual patient's needs. This requirement is clearly demonstrated by comparing Cases 4 and 5. Case 4 was an elderly, slightly hypertensive woman, whose ventriculogram showed slight frontal atrophy. She was completely relieved after eight focal coagulations in each frontal lobe; this was slightly more damage than she really required, but only slightly more. She lost a little of her intellectual capability, but she is extremely happyif anything, rather too happy. Case 5, on the other hand, was a fit man of 46. He was relieved of his symptoms, with a significant increase in his intellectual capability, by the production of 16 focal lesions in each frontal lobe. That is, he had twice as much damage produced in the frontal lobes as the elderly woman.

The longest time we have left electrodes in the brain is seven months. This period gives adequate scope for estimating the degree of interference needed for a particular patient, but there is no reason why electrodes should not be left in position longer if the physician thinks it necessary.

During the two years since this procedure was introduced, the results of treatment have been strikingly good in the three successful cases in terms of intellectual capacity, emotional balance, and social adjustment. In the four patients now under treatment, the results are also very promising. The possibility of giving relief from otherwise intractable suffering, and the control that can be exercised over the site and extent of cerebral lesions are the justification for this preliminary report. Now that the technical difficulties have been overcome and the risks of the procedure can be balanced against its advantages, it is intended to test its value more exactingly in a larger selection of patients.

\section{Summary}

A method has been developed for obtaining greater precision in the control of the site, size, and duration of lesions in frontal leucotomy. It involves the introduction of large numbers of small, chronically indwelling, gold intracerebral electrodes in the 
frontal lobes. A temporary leucotomy can be achieved by electrical polarization of these electrodes; where relief is obtained a permanent selective leucotomy can be performed by electro-coagulation using direct current.

The results in the first five cases are briefly described.

The effects of the procedure can be observed while the patients are leading a normal life as well as in hospital.

No difficulties have been encountered with infection, insertion trauma, or electrode reaction. The patients tolerate the implantation well for periods of several months.

We should like to express our deep appreciation of the encouragement given to us by Professor F. L. Golla, who was the Director of the Burden Neurological Institute when this work was begun.

We are immeasurably indebted to Dr. W. Grey Walter for the help, advice, and stimulating discussions which promoted this work.

We should like to thank Mr. G. L. Alexander and Mr. A. Hulme for their sympathetic interest in our work, and for performing the operation on two of the patients.

We are grateful to Dr. C. W. Sem-Jacobsen and Dr. Arne Torkildsen for a patient and helpful demonstration in Gaustad Sykehus, Oslo, Norway, of their method of introducing multiple fine wire electrodes into the brain. This was arranged through the good offices of the Air Research and Development Command of the United States Air Force.

Others of our colleagues whose help we gratefully acknowledge are Dr. V. O. G. Smyth for psychological testing of the patients; Miss Dorothy Wardley and her nursing staff for their understanding care of their patients; of Mr. Arthur Winter for assistance in the experimental $\underset{0}{\stackrel{a}{a}}$ work; and Mr. W. J. Warren and Mr. H. Pask for the design and construction of special equipment.

Finally we should like to thank the various manufacturers who have supplied the materials, especially $F$. D. Sims Ltd., Ramsbottom, Lancs., who have been responsible for enamelling of electrode wire, and Dr. Franklin D. Offner who lent us the recording instrument used to observe the physiological responses of the patients.

\section{REFERENCES}

Chang, H.-T. (1959) Handbook of Physiology, p. 299. American

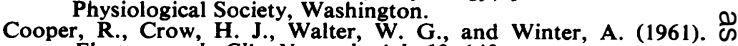
Electroenceph. Clin. Neurophysiol., 13, 143.

Dodge, H. W. Jr., Holman, C. B., Sem-Jacobsen, C. W., Bickford, Fischer. (1953). Proc. Mayo Clin., 28, 147. Fischer, G., Sayre, G. P., and Bickford, R. G. (1957). Ibid, 32, 14. Fleming, D. G. (1957). Electroenceph. Clin. Neurophysiol., 9, 551 . Freeman, W. (1948). Lancet, 2, 371 .

Grantham, E. G. (1951). J. Neurosurg., 8, 405.

Greenblatt, M., and Solomon, H. C. (1952). Amer. J. Psychiat., 109, 262 .

Loucks, R. B., Weinberg, H., and Smith, M. (1959). Electroenceph.

Clin. Neurophysiol., 11, 823.
MacIntrye, W. J., Bidder, T. G., and Rowland, V. (1959). In Proceedings of the First National Biophysics Conference, Columbus, Ohio, 1957, Vol. XXVII, p. 723. Yale University Press, New York.

McKissock, W. (1951). Lancet, 2, 91.

Pool, J. L. (1949). Proc. roy. Soc. Med., 42, Suppl. p. 1.

Scoville, W. B. (1949). J. Neurosurg., 6, 65. Spiegel, E. A., and Wycis, H. T. (1949). Proc. roy. Soc. Med., 48
Suppl. p. 84 .

Thorpe, F. T., and Hardman, J. (1952). J. ment. Sci., 98, 389.

White, J. C., Sweet, W. H., and Hackett, T. P. (1959). Trans. Amer neurol. Ass., pp. 88-92, and (1960) Arch. Neurol. Psychiat., 317. Whitty, C. W. M., Duffield, J. E., Tow, P. M., and Cairns, H. (1952
Lancet, 1, 475 . 